Artigo original

Hegemonia - Revista Eletrônica do Programa de Mestrado em Direitos Humanos,

Cidadania e Violência/Ciência Política do Centro Universitário Unieuro

ISSN: $1809-1261$

UNIEURO, Brasília, número 25 (Especial), 2018, pp. 7-25.

Recebido em: 23/03/2018

Avaliado em: 15/04/2018

Aprovado em: 10/05/2018

\title{
NANOSISTEMAS LIPÍDICOS E POLIMÉRICOS PARA APLICAÇÃO TÓPICA NO TRATAMENTOD FERIDAS
}

\author{
Jaqueline Rodrigues da Silva ${ }^{1}$ e Franciele de Matos da Silva ${ }^{2}$
}

Resumo: A engenharia de tecido ou bioengenharia tem avançado em estudos e propostas eficazes para o tratamento clínico de diversas doenças da pele e em questões estéticas. Apesar disto, há uma expressiva limitação de fármacos e formas farmacêuticas que superem as dificuldades impostas pelo microambiente de uma pele danificada por feridas e ou lesões. Novas formas farmacêuticas e sistemas de entrega de fármacos nanoestruturados estão em estudos, como propostas tecnológicas que promovam uma melhor eficácia na ação tópica e sistêmica dos fármacos. Estruturas lipídicas e poliméricas com tamanho nanométrico são aplicadas isoladamente ou em associação com biomembranas e desenvolvidas para atuarem como umas das soluções no tratamento de peles traumatizadas por lesões cutâneas, reduzindo a toxicidade do fármaco e melhorando sua permeação através das camadas da pele. A evolução nas tecnologias e matérias primas para o desenvolvimento destes sistemas, descritos nesta revisão; demonstra o avanço na busca de novos métodos de tratamento com o uso de lipossomas e nanoesferas para carregar macromoléculas lipossolúveis e hidrossolúveis, manter o controle microbiano e a homeostase nas regiões de feridas crônicas e promover uma reparação tecidual mais rápida e indolor.

Palavras Chaves: Feridas, Nanobiotecnologia, Nanopartículas, Transdérmico.

Abstract: Tissue engineering or bioengineering has advanced in studies and proposals effective for the clinical treatment of various skin diseases and aesthetic issues. Despite this, there is an expressive limitation of drugs and pharmaceutical forms that overcome the difficulties imposed by the microenvironment of a skin damaged by wounds and or lesions. New pharmaceutical forms and nanostructured drug delivery systems are under study as technological proposals that promote better efficacy in the topical and systemic action of the drugs. Nano-sized lipid and polymeric structures are applied alone or in association with biomembranes and developed to act as one of the solutions in the treatment of skin traumatized by skin lesions, reducing drug toxicity and improving its permeation through the

\footnotetext{
${ }^{1}$ Doutora em Ciências Biológicas e docente da Universidade de Brasília.

2 Mestranda em Engenharia Biomédica pela Universidade de Brasília.
} 
Artigo original

Hegemonia - Revista Eletrônica do Programa de Mestrado em Direitos Humanos, Cidadania e Violência/Ciência Política do Centro Universitário Unieuro

ISSN: 1809-1261

UNIEURO, Brasília, número 25 (Especial), 2018, pp. 7-25.

layers of the skin. The evolution in technologies and raw materials for the development of these systems, described in this review; demonstrates the advances in the search for new treatment methods with the use of liposomes and nanospheres to carry liposoluble and water soluble macromolecules, to maintain microbial control and homeostasis in the regions of chronic wounds and to promote a quicker and painless tissue repair.

Key words: Wounds healing, Nanobiotechnology, Nanoparticles, Skin drug delivery.

Introdução

A aplicação biomédica de sistemas de carregamento de fármacos nanoestruturado é uma das ferramentas para o tratamento de diversas doenças dermatológicas com o desenvolvimento de novas estruturas cujo objetivo é facilitar a aplicação e melhor a ação local de diversos fármacos.

O mercado de transdermicos é ainda muito limitado, com poucos fármacos em uso, os quais na sua maioria são moléculas de tamanhos pequenos e de caráter lipofílicos com a finalidade de superar as dificuldades impostas pelas barreiras da pele como: (i) dificuldade de transportar macromoléculas terapêuticas através das camadas de pele e (ii) a baixa permeabilidade do extrato córneo a moléculas e fármacos hidrossolúveis[1]. Comparando com as formas farmacêuticas e os sistemas convencionais de entrega de fármacos como géis, cremes e loções entre outros [2,3], as nanoestruturas vem atraindo muita atenção da indústria farmacêutica por melhorar a ação local ou sistêmica do fármaco e, por conseguinte sua biodistribuição.

Apesar do tamanho nanométrico das nanopartículas garantir a capacidade de adesividade quando em contato com superfícies [4], a entrega de fármacos na epiderme e derme usando sistemas nanoparticulados está em constante questionamento. Por outro lado, quando existe alguma lesão na pele, como envelhecimento, doenças ou feridas na pele (venosa, pé diabético e úlceras), observa-se um aumento potencial para aplicação desses 
Artigo original

Hegemonia - Revista Eletrônica do Programa de Mestrado em Direitos Humanos,

Cidadania e Violência/Ciência Política do Centro Universitário Unieuro

ISSN: 1809-1261

UNIEURO, Brasília, número 25 (Especial), 2018, pp. 7-25.

sistemas [5]. Um nanosistema é capaz de transportar fármacos hidrossolúveis e lipossolúveis através das camadas da pele ou promover a liberação gradativa dentro da região da pele acometida por feridas.

A engenharia de tecido é uma das áreas em evolução para o uso dessas partículas, como carregadores para entrega de fármacos, fatores de crescimento celular e pró angiogênicos, aplicados isoladamente ou como formas farmacêuticas tópicas associadas a coberturas (curativos ou "dressing") [6].

Dessa maneira esta revisão é baseada descrever os sistemas nanoparticulados de composição lipídicas e poliméricas desenvolvidas para aplicação tópica em peles acometidas por lesões às quais facilitam as terapias locais.

Feridas de Pele

A pele é o maior órgão do nosso corpo, que corresponde cerca de 16\% do peso corporal, considerada uma barreira de proteção com função sensorial e de regulação [7]; e frequentemente é lesionada por feridas ou traumas físicos.

Alterações dermatológicas, como doenças de pele, infecções e reações cutâneas associadas a doenças crônicas, podem levar ao desenvolvimento das feridas crônicas [8]. A área da pele acometida por uma ferida crônica pode ser descrita como um ambiente favorável a inflamações recorrentes, não regeneração da epiderme, fibroblastos e matriz extracelular não funcionais, agiogênse reduzida, níveis de protease aumentado e colonização bacteriana $[9,10]$.

O tratamento de feridas sempre foi baseado na manutenção da região afetada sem a presença de microorganismos e seca, para que ocorra a reparação tecidual e cicatrização [11], 
Artigo original

Hegemonia - Revista Eletrônica do Programa de Mestrado em Direitos Humanos, Cidadania e Violência/Ciência Política do Centro Universitário Unieuro

ISSN: $1809-1261$

UNIEURO, Brasília, número 25 (Especial), 2018, pp. 7-25.

o grande arsenal de produtos como pomadas géis e soro vem passando por avanços científicos e tecnológicos ao longo dos anos, com o objetivo de reduzir as lesões e ocasionar um melhor conforto aos pacientes [12,13].

O curativo é uma forma de tratamento de feridas cutâneas, desenvolvido para atuar de forma dinâmica, na evolução das fases da cicatrização (homeostase, inflamatória, proliferativa e de remodelação) [14]. Para esta necessidade, curativos contendo antibióticos, antibacterianos, antissépticos, antimicrobianos, enzimas proteolíticas e fatores de crescimento entre outros estão disponíveis no mercado, além de produtos direcionados á proteção da pele contra as lesões (desbridamento químico, enzimático, autolítico ou mecânico e coberturas) [15]. As formas farmacêuticas mais encontradas são à base de pomadas e óleos como: curativo com Sulfadiazina de prata (Dermazine ${ }^{\circledR}$ e Pratazine $\left.{ }^{\circledR}\right)$, curativos com pomada Enzimática - Colagenase (Iruxol®, Kollagenase ${ }^{\circledR}$, Santyl $\left.{ }^{\circledR}\right)$, curativos com Ácido Graxos Essenciais (AGE) (Agederm ${ }^{\circledR}$, Ativoderme ${ }^{\circledR}$, Dersani ${ }^{\circledR}$ ), curativos com hidrocoloides (Comfeel ${ }^{\circledR}$. Duoderm ${ }^{\circledR}$, Hydrocoll ${ }^{\circledR}$, Tegasorb $\left.\AA\right)$, curativos com hidrogel (Duoderm Gel®, Hydrosob), curativos com alginato de Cálcio (Algoderm ${ }^{\circledR}$, Curasorb ${ }^{\circledR}$, Sorbalgon ${ }^{\circledR}$, Tegagen () , curativo a vácuo (V.A.C. $\left.{ }^{\circledR}\right)[16,17]$.

Baseados nos curativos, novas plataformas para o tratamento das alterações da pele, que consigam resultados mais eficazes e robustos estão em crescente avanço pela introdução da bioengenharia e engenharia de tecidos [18]. Terapias que mimetizem a pele humana com a finalidade de fechar a ferida, controlar a perda de fluidos e exibir propriedades como durabilidade, elasticidade e histocompatibilidade são desenvolvidas com a utilização dos chamados biomateriais inteligentes constituídos pela combinação de sistemas para entrega específica de fármacos ou moléculas bioativas associados a coberturas (membranas e “scaffolds") [19,20,21]. 
Artigo original

Hegemonia - Revista Eletrônica do Programa de Mestrado em Direitos Humanos, Cidadania e Violência/Ciência Política do Centro Universitário Unieuro

ISSN: 1809-1261

UNIEURO, Brasília, número 25 (Especial), 2018, pp. 7-25.

Os nanosistemas aplicados no tratamento de feridas crônicas podem ser de materiais diversos e específicos para cada fase da cicatrização da ferida, na revisão publicada por Hamdan e colaboradores [22], é descrito os avanços nas pesquisas e nos muitos estudos clínicos realizados e em andamentos.

Lipossomas e Sistemas Nanoparticulados Lipídicos.

Lipossomas são vesículas lipídicas, descrita por Bangham e colaboradores [23] em 1965 que demonstraram a formação espontânea de uma estrutura vesicular após a mistura dos lipídios com água. Esta estrutura vesicular foi inicialmente usada como modelo de membrana e desde a década de 70 vem sendo estudada para entrega de fármacos [24]. A característica anfipática dos fosfolipídios facilita o carregamento de fármacos lipossolúveis e hidrossolúveis em formulações lipossomais que abrange fármacos para o tratamento do câncer, antibacterianos, antifúngicos, e imunomodulares, entre outros [25,26]. De maneira geral, são classificados em três grupos de acordo com a morfologia apresentada: (i) vesículas grandes multilamelares (MLVs) com diâmetros na faixa de 400 a 3500 nm; (ii) vesículas grandes unilamelares (LUVs), estes lipossomas possuem diâmetros que variam de 100 a 1000 nm e (iii) vesículas pequenas unilamelares (SUVs), são as que apresentam o menor limite possível de tamanho para vesículas de fosfolipídios (diâmetros de 20 a $50 \mathrm{~nm}$ ) [3]

Preparações lipossomais para aplicações em humano são administradas por diferentes vias e formas [27] algumas já a venda no mercado [28] e estão em constante processo de evolução decorrentes dos novos materiais e métodos para obtenção destes sistemas. Um exemplo são os estudos e pesquisas desenvolvidas por diferentes grupos para sua aplicação tópica com o objetivo de melhorar a entrega de fármacos nas diferentes 
Artigo original

Hegemonia - Revista Eletrônica do Programa de Mestrado em Direitos Humanos, Cidadania e Violência/Ciência Política do Centro Universitário Unieuro

ISSN: 1809-1261

UNIEURO, Brasília, número 25 (Especial), 2018, pp. 7-25.

camadas da pele (epiderme, derme, e hipoderme) [29, 30,31,32,33,34] Sabe-se que o objetivo dos sistemas de liberação e entrega é obter uma melhor eficácia terapêutica do fármaco por meio da redução da toxicidade e do aumento da sua eficiência, baseado nas mudanças na farmacocinética e fisiologia do mesmo.

Modificações nas formas farmacêuticas tradicionais para uso tópico, como soluções, cremes, géis pastas entre outros vem sendo desenvolvidos a fim de melhorar a permanência de preparações líquidas na área afetada, as quais incluem emulsões, microemulsões, formulações lipossomais (convencionais), transferossomos, niossomos, ethossomos, nanopartículas lipídicas sólidas, microesferas e nanoesferas [35,36].

Segundo revisão de. Nounou e colaboradores a descrição do uso de lipossomas para administração tópica foi inicialmente apresentada por Messei e Gulasekharam que avaliou o aumento da deposição em pele de coelho de um corticosteroide (triancinolona) após sua encapsularão em lipossomas [35]. Estudos com lipossomas convencionais para aplicação na pele, promovido por Touitou e colaboradores no final da década de 90, concluíram que não ocorre a penetração destas estruturas na pele e sim sua permanência na camada superior do extrato córneo [37]. Assim, novas estruturas foram construídas usando o mesmo conceito vesicular de mistura de lipídios e água, mas com características "deformáveis" ou “elásticas”[34,35, 38,39]

Os lipossomas deformáveis e etossomos (Transfersomes $\left.{ }^{\circledR}\right)$ são considerados a primeira geração de vesículas elásticas e foram descritas por Cevec \& Blume em 1992 [40], na mesma década van den Bergh e colaboradores [41] utilizando surfactantes descreveram os niossomos. Os dois sistemas com características vesicular são preparadas com compostos que facilitam e melhoram as interações moleculares entre vesícula, fármaco e camadas da pele, para isso foi usado o etanol nas formulações de etossomos; e os surfactantes aniônicos 
Artigo original

Hegemonia - Revista Eletrônica do Programa de Mestrado em Direitos Humanos, Cidadania e Violência/Ciência Política do Centro Universitário Unieuro

ISSN: 1809-1261

UNIEURO, Brasília, número 25 (Especial), 2018, pp. 7-25.

nos nissomos; e assim estudos para melhoria da solubilidade e estabilidade do ácido retinóico em vesículas catiônicas [42], retenção e permeação cutânea de paramomicina lipossomal para tratamento de lesões cutâneas de leshimaniose [43], vesículas carregadas de fármacos fotosensibilizantes para terapia fotodinâmica de lesões de pele $[44,45,46]$, lipossomas deformáveis contendo metotrexato [47] para aplicação tópica de um antineoplásico, lipossomas elásticos para tratamento de doenças sistêmicas pela via transdermal [48] foram realizados ao longo dos anos.

No inicio dos anos 90, partículas, emulsões e lipossomas foram combinadas para o desenvolvimento das chamadas nanopartículas lipídicas sólidas (NLS) [49,50] que apresentam núcleo lipídico com capacidade de melhorar a liberação de fármacos em mucosa [51]. Sua composição e estrutura formam um filme sobre a pele que aumenta a penetração de moléculas ou aumenta a quantidade de água nas camadas superior da epiderme, promovendo efeitos sistêmicos após sua administração tópica e um melhor uso de nanopartículas em produtos para esta via de administração [52,53].

Recentemente foi descrito que a incorporação de lipídios líquidos, a temperatura ambiente, como um dos constituintes das nanopartículas lipídicas, leva a formação de uma nova classe de carregadores com tamanho nanométrico, os chamados carregadores lipídicos nanoestruturados (NCL), onde a fase líquida pode ser a superfície da nanopartícula ou pode está numa matriz sólida; e levando uma nova forma para aplicações dermatológicas $[54,55,56]$. É descrito por Honeywell-Nguyen e colaboradores $[57,58]$ dois mecanismos que explica o melhoramento da entrega de fármacos por estes sistemas: (i) vesículas intactas entram no extrato córneo carregando moléculas para o interior da pele e (ii) vesículas podem aumentar a penetração pela modificação da sua estrutura vesicular de bicamada em lipídio lamelar (membrana celular). 
Artigo original

Hegemonia - Revista Eletrônica do Programa de Mestrado em Direitos Humanos, Cidadania e Violência/Ciência Política do Centro Universitário Unieuro

ISSN: 1809-1261

UNIEURO, Brasília, número 25 (Especial), 2018, pp. 7-25.

Sistemas Nanoparticulados Poliméricos

Nanopartículas poliméricas são definidas com estruturas sólidas e esféricas, com tamanhos variando de 10 a $1000 \mathrm{~nm}$, compostas de polímeros naturais e/ou sintéticos com capacidade de carregar e entregar fármacos e moléculas bioativas variadas, desde macromoléculas biológicas, vacinas e fármacos hidrossolúveis e lipossolúveis [59,60].

O termo nanopartícula é coletivo para qualquer tipo de polímero, mas foi usado no final dos anos 80 por Couvrier [61] para especificar nanoesferas e nanocápsulas como sistemas coloidais de: (i) matriz polimérica, que carrega fármaco na superfície ou misturado na matriz - nanoesferas; e (ii) sistema vesicular que confinam substâncias em um núcleo ou centro (óleo ou água) cercado por uma casca polimérica - nanocápsulas.

Diferentes tipos de polímeros são estudados no desenvolvimento de sistemas para entrega de fármacos nanoparticulados, suas misturas, degradação, biocompatibilidade, toxicidade, aplicações biológicas e métodos de preparações são descritas na literatura científica e revisadas recentemente [62, 63,64]. No desenvolvimento desses sistemas, os polímeros sintéticos como poli etileno glicol (PEG), poli caprolactona (PCL). ácido poliláctico (PLA), ácido poli-glicólico e seus copolímeros $[65,66,67]$ são os mais aplicados e estudados nos testes in vitro e in vivo.

O copolímero PLGA (Poli (láctico-ácido co-glicólico) é o polímero sintético biodegradável mais utilizado e aprovado pelas agências americana (FDA) e europeia (EMA) para aplicação em animais e humano; sua hidrólise leva a produção de metabólitos monoméricos que são metabolizados facilmente pelo organismo; tornando seus produtos de degradação atóxicos, biodegradáveis e biocompatíveis [68,69]. 
Artigo original

Hegemonia - Revista Eletrônica do Programa de Mestrado em Direitos Humanos, Cidadania e Violência/Ciência Política do Centro Universitário Unieuro

ISSN: 1809-1261

UNIEURO, Brasília, número 25 (Especial), 2018, pp. 7-25.

A quitosana é o polímero natural mais estudado para o desenvolvimento de nanosistemas coloidais, aplicados na bioengenharia e medicina regenerativa. É um polissacarídeo de origem animal, biocompatível para uso em humano, não tóxico e de grande aplicação na farmácia e medicina como matéria prima na produção de coloides e membranas usados para liberação controlada de fármacos [70,71,72].

Os estudos utilizando polímeros na produção destes sistemas tem na sua maioria a aplicação pela via endovenosa e intramuscular, para que carreguem mais fármacos pela circulação - reduzindo a toxicidade sistêmica; e com as modificações na superfície das partículas possam direcioná-las ao local de ação $[73,74]$. Nas novas pesquisas biomédicas, impulsionadas pela medicina regenerativa e engenharia de tecidos com alvos na regeneração de tecidos e órgãos, promoveu a utilização destes sistemas poliméricos combinados ou não com células (tecidos) matrizes e suportes ("scaffolds", membranas e hidrogeis), para favorecer uma liberação controlada de maneira espacial e temporal [75] melhorando suas aplicações tópicas.

É sabido que o reparo da pela danificada por ferida crônica é um processo biologicamente complexo, tendo necessidade de fechar a região afetada. Estudos recentes têm levantado o interesse de materiais poliméricos biodegradáveis, biocompatíveis e bioreabsorvível, que podem ser absorvidos in situ, e metabolizados pelo hospedeiro, tendo como inspiração as abordagens convencionais utilizam o modelo de cobertura da área afetada. Polímeros de fontes naturais, como colágeno, gelatina proteínas ou polissacarídeos são utilizados como matéria prima para estes propósitos. [76].

Os hidrogéis, uma estrutura tridimensional hidrofílica formada por uma rede polimérica capaz de absorver uma grande quantidade de água ou fluidos biológicos [77], são uma dessas plataformas, onde nanosistemas lipídicos são incorporados dentro da malha 
Artigo original

Hegemonia - Revista Eletrônica do Programa de Mestrado em Direitos Humanos, Cidadania e Violência/Ciência Política do Centro Universitário Unieuro

ISSN: 1809-1261

UNIEURO, Brasília, número 25 (Especial), 2018, pp. 7-25.

polimérica favorecendo a estabilidade e liberação de antibióticos, antifúngicos, antineoplásicos, antioxidantes e antiinflamatório [787,79].

Coberturas a base de quitosana devido a sua natural atividade antibactericida, podem também carregar nanopartículas lipídicas e de PLGA, atuando nas infecções por biofilmes bacterianos, na liberação sustentada de fatores de crescimento celular ou de analgésicos $[80,81,82,83,84]$.

Perspectivas

Apesar da evolução dos sistemas nanoparticulados para entrega de fármaco ocorrido ao longo dos anos, a aplicação na clínica ainda é muito reduzida. Sejam por dificuldades no processo de fabricação, de transposição do laboratório para a indústria ou pelos custos inerentes as novas tecnologias.

A associação dos diferentes tipos de nanosistemas aqui descritos nessa revisão, que possuem capacidade de atuar efetivamente no microambiente de uma ferida crônica, leva a necessidade de promover uma aplicação mais efetiva dessa tecnologia; com objetivos de formular novos produtos com capacidade de manter uma menor quantidade de microorganismo na área afetada, pelo carregamento de antimicrobiano para ação local; e ao mesmo tempo manutenção da umidade do tecido, suportando a reparação do mesmo.

Estudo para a utilização de bases como géis e biomembranas, matérias e excipientes de origem natural, fototerapias em associação com as partículas lipídicas e poliméricas descritas é o que tem movimentado os pesquisadores e as indústrias numa nova geração de tratamento curativo para doenças de pele. 
Artigo original

Hegemonia - Revista Eletrônica do Programa de Mestrado em Direitos Humanos,

Cidadania e Violência/Ciência Política do Centro Universitário Unieuro

ISSN: $1809-1261$

UNIEURO, Brasília, número 25 (Especial), 2018, pp. 7-25.

Referências Bibliográficas

1. Szunerits S, Boukherroub R., Heat: A Highly Efficient Skin Enhancer for Transdermal Drug Delivery, In Front Bioeng Biotechnol, 2018, vol. 6, p. 15.

2. Loyd V. Allen Jr., Nicholas G. Popovich; Howard C. Ansel, In Formas Farmacêtiticas e Sistemas de Liberação de Fármacos, 2013, $9^{a}$ Edição, Editora Artmed, São Paulo, Brasil.

3. J de Leeuw, HC de Vijlder,P Bjerring, HAM Neumann, Liposomes in dermatology today In Journal European Academy of Dermatology and Venereology (JEADV), 2009, Vol. 23 , pp 505-516.

4. Souto, E.B., Müller, R.H., Cosmetic features and applications of lipid nanoparticles (SLN, NLC). In International Journal of Cosmetic Science, 2008, vol. 30, n. 3, pp. 157-165.

5. Makhmalzade BS, Chavoshy F. Polymeric micelles as cutaneous drug delivery system in normal skin and dermatological disorders, In Journal Adv. Pharm.Technol., 2008, vol. 9(1), pp.2-8.

6. J.C. Chappell, J. Song, C.W. Burke, A.L. Klibanov, R.J. Price, Targeted delivery of nanoparticles bearing fibroblast growth factor-2 by ultrasonic microbubble destruction for therapeutic arteriogenesis, In Small, 2008, Vol. 4, pp. 1769-1777.

7. Schmidt MI, Duncan BB, Azevedo e Silva G, Menezes AM, et.al.. In The Lancet, 2011, vol. 377, n. 11, Issue 9781, p. 1.949-1.

8. Ramos, A.,In Ebook Manifestações dermatológicas do Diabetes, 2015.

9. Brem, H.; Tomic-Canic, M. Cellular and molecular basis of wound healing in diabetes. In Journal. Clinical. Investigation. 2007, Vol. 117, pp.1219-1222.

10. Pastar, I.; Stojadinovic, O.; Yin, N. C.; Ramirez, H.; Nusbaum, A. G.; Sawaya, A.; Patel, S. B.; Khalid, L.; Isseroff, R. R.; Tomic-Canic, M.Epithelialization in wound healing: a comprehensive review. In Adv.Wound Care, 2014, vol. 3, pp. 445-464.

11. Tavares W.S., Silva R.S. Curativos utilizados no tratamento de queimaduras: uma revisão integrativa. In Revista Bras Queimaduras. 2015, vol. 14 (4), pp. 300-6.

12. Mandelbaum, S.H., Di Santis, E.P.; Mandelbaum, M.H.S., Cicatrização: conceitos atuais e recursos auxiliares - Parte I, In Anais Bras. Dermatol., 2003, vol.78, n.4, pp.393-408.

13. Dealey C. In Cuidando de Feridas: um guia para as enfermeiras. São Paulo, Atheneu Editora, $2^{a}$ edição, 2001, capítulo 3: p. 49-65, capítulo 4: p. 68-89; cap. 9: p. 200-207. 
Artigo original

Hegemonia - Revista Eletrônica do Programa de Mestrado em Direitos Humanos,

Cidadania e Violência/Ciência Política do Centro Universitário Unieuro

ISSN: $1809-1261$

UNIEURO, Brasília, número 25 (Especial), 2018, pp. 7-25.

14. Rebecca S. Howell-Jones, Patricia E. Price, Anthony J. Howard, David W. Thomas, Antibiotic prescribing for chronic skin wounds in primary care. In Wound Repair and regeneration, 2006, vol.14 (4), p. 387-39.

15. Mandelbaum S.H., Di Santis E.P., Mandelbaum M.H.S. Cicatrização: Conceitos atuais e recursos auxiliares. Parte II. In Anais Bras Dermatol. 2003, vol. 78(5), pp. 525- 42.

16. Morgan D.A. Wound dressing: principals and types of dressings. In: Formulary of Wound Managment Products: a guide for health care staff, 6 th ed. Haslemere, Surrey: Euromed Communications; 1994, p.64-73.

17. Johnson A. Journal of Wound Care Nursing. Dressing for deep wounds. In Nurs Time, 1992, vol. 88(4), pp. 56-8.

18. J.C. Chappell, J. Song, C.W. Burke, A.L. Klibanov, R.J. Price, Targeted delivery of nanoparticles bearing fibroblast growth factor-2 by ultrasonic microbubble destruction for therapeutic arteriogenesis, In Small, 2008, vol. 4, pp.1769-1777.

19. Korrapati, P. S.; Karthikeyan, K.; Satish, A.; Krishnaswamy, V.R.; Venugopal, J. R.; Ramakrishna, S. Recent advancements in nanotechnological strategies in selection, design and delivery of biomolecules for skin regeneration. In Mater. Sci. Eng., 2016, vol. 67, pp. $747-765$.

20. Veves, A.; Falanga, V.; Armstrong, D. G.; Sabolinski, M. L.Graftskin, A human skin equivalent, is effective in the management of noninfected neuropathic diabetic foot ulcers a prospective randomized multicenter clinical trial. In Diabetes Care, 2001, vol. 24, pp. $290-295$.

21. Driver, V. R.; Lavery, L. A.; Reyzelman, A. M.; Dutra, T. G.; Dove, C. R.; Kotsis, S. V.; Kim, H. M.; Chung, K. C. A clinical trial of Integra Template for diabetic foot ulcer treatment. Wound Repair Regen. 2015, vol. 23, pp. 891-900

22. Hamdan S., Pastar I., Drakulich S., Dikici E., et. al., Nanotechnology-Driven Therapeutic Interventions in Wound Healing: Potential Uses and Applications, In ACS Cent. Sci., 2017, vol. 3(3), pp. 163-175.

23. Bangham A.D., Standish M.M., Watkins J.C., Diffusion of univalent ions across the lamellae of swollen phospholipids. In Journal of Molecular Biology, 1965; vol. 13, pp.238252.

24. Schmid M.H., Korting H.C., Therapeutic progress with topical liposome drugs for skin disease. In Advanced Drug Delivery Review, 1996; vol.18, pp 335-342. 
Artigo original

Hegemonia - Revista Eletrônica do Programa de Mestrado em Direitos Humanos,

Cidadania e Violência/Ciência Política do Centro Universitário Unieuro

ISSN: $1809-1261$

UNIEURO, Brasília, número 25 (Especial), 2018, pp. 7-25.

25. Morille M, Passirani C, Dufort S, Bastiat G, Pitard B, Coll JL, et al. Tumor transfection after systemic injection of DNA lipid nanocapsules. In Biomaterials, 2011, vol. 32, pp.2327-33.

26. Lin-Ai Tai, Pi-Ju Tsai, Yu-ChaoWang, Yu-JingWang, et. al., Thermosensitive liposomes entrapping iron oxide nanoparticles for controllable drug release, In Nanotechnology, 2009, vol. 20, pp.135101 (9pp).

27. Nounou, Mohamed \& El-Khordagui, L.K. \& Khalafallah, Nawal \& A Khalil, Said, Liposomal Formulation for Dermal and Transdermal Drug Delivery: Past, Present and Future. In Recent patents on drug delivery \& formulation, 2008, vol.2, pp 9-18.

28. Mehran Alavi, Naser Karimi, and Mohsen Safaei. In Adv Pharm Bull. 2017, vol.7(1), pp. 3-9.

29. Williams, A.C., Barry, B.W., 1991. The enhancement index concept applied to terpene penetration enhancers for human skin and model lipophilic (oestradiol) and hydrophilic (5-fluorouracil) drugs. In International of Journal Pharma., 1991, vol. 74, pp. 157-168.

30. Schreier, H., Bouwstra, J., 1994. Liposomes and niosomes as topical drug carriers: dermal and transdermal drug delivery. In Journal of Control Release, 1994, vol. 30, pp. 115.

31. Kirjavainen, M., Urtti, A., Jaaskelainen, I., Suhonen,T.M., Paronen, P.,ValjakkaKoskela, R., Kiesvaara, J., Monkkonen, J., Interaction of liposomes with human skin in vitro-the influence of lipid composition and structure. Biochim. Biophys. Acta, 1996, vol.1304, pp. 179-189.

32. Bouwstra, J.A., Honeywell-Nguyen, P.L., Skin structure and mode of action of vesicles. In. Adv. Drug. Deliv. Rev., 2002, vol. 54, S41-S55.

33. El Maghraby, G.M.,Williams, A.C., Barry, B.W., 2006. Can drug-bearing liposomes penetrate intact skin? J. In Pharm. Pharmacol., 2006, vol. 58, pp. 415-429.

34. Mustafa M.A. Elsayed, Ossama Y. Abdallah, Viviane F. Naggar, Nawal M. Khalafallah Deformable liposomes and ethosomes: Mechanism of enhanced skin delivery, In International Journal of Pharmacentics, 2006, vol. 322, pp.60-66.

35. Mohamed I. Nounoua,b, Labiba K. El-Khordaguib, Nawal A. Khalafallahb and Said A. Khalilb. Liposomal Formulation for Dermal and Transdermal Drug Delivery: Past, Present and Future. In Recent Patents on Drug Delivery \& Formulation, 2008, vol.2, pp. 9-18. 
Artigo original

Hegemonia - Revista Eletrônica do Programa de Mestrado em Direitos Humanos,

Cidadania e Violência/Ciência Política do Centro Universitário Unieuro

ISSN: 1809-1261

UNIEURO, Brasília, número 25 (Especial), 2018, pp. 7-25.

36. Roberts M.S., Mohammed Y, Pastore M.N., Namjoshi S, Yousef S, Alinaghi A, Haridass I.N., Abd E, Leite-Silva V.R., Benson H, Grice J.E. Topical and cutaneous delivery using nanosystems. Control Release. 2017, vol. 247, pp. 86-105.

37. Touitou, E., Dayan, N., Bergelson, L., Godin, B., Eliaz, M., Ethosomes—novel vesicular carriers for enhanced delivery: characterization and skin penetration properties. In J. Control. Release, 2000, vol. 65, pp. 403-418

38. Vyas S, Uchegbu IF. Non ionic surfactant based vesicles (niosomes) in drug delivery. In Int J Pharm, 1998, vol.172, pp. 33-70.

39. Montanari J., Cristina Maidana C., Esteva M.I., et.al. S, Sunlight triggered photodynamic ultradeformable liposomes against Leishmania braziliensis are also leishmanicidal in the dark, In Journal of Controlled Release 2010, vol.147(3), pp. 368-76.

40. Cevc, G., Blume, G., Lipid vesicles penetrate into intact skin owing to the transdermal osmotic gradients and hydration force. In Biochim. Biophys. Acta, 1992. vol. 1104, pp. 226232.

41. van den Bergh, B.A., Bouwstra, J.A., Junginger, H.E., Wertz, P.W., 1999. Elasticity of vesicles affects hairless mouse skin structure and permeability. In J. Control. Release, 1999, vol. 62, pp. 367-379.

42. Aust D, Judd C, Wilmott J. Stability enhancement of retinol through encapsulation in cationic vesicles. In J Cosmetic Si 2000, vol, 51(1), pp. 52-53.

43. Ferreira LS, Ramaldes GA, Nunan EA, Ferreira LA. In vitro skin permeation and retention of paromomycin from liposomes for topical treatment of the cutaneous leishmaniasis. In Drug Dev Ind Pharm, 2004, vol.30(3), pp. 289-96.

44. Velloso N.V., Muehlmann L.A., Longo J.P.F., da Silva J.R., Zancanela D.C., et al. Aluminum-Phthalocyanine Chloride-Based Photodynamic Therapy Inhibits PI3K/Akt/Mtorpathway in Oral Squamous Cell Carcinoma Cells In Vitro. In Chemotherapy, 2012, vol. 1 (5), p.107.

45. Di Venosa G., Hermida L., Batlle A., Fukuda H., et.al., Characterisation of liposomes containing aminolevulinic acid and derived esters, In Journal of Photochemistry and Photobiology B: Biology, 2008, vol.92, pp. 1-9.

46. Pashkovskaya A., Kotova E., Zorlu Y., Dumoulin F., Ahsen V., Agapov I., Antonenko Y., In Langmuir, 2010, vol. 26(8), pp.5726-5733. 
Artigo original

Hegemonia - Revista Eletrônica do Programa de Mestrado em Direitos Humanos,

Cidadania e Violência/Ciência Política do Centro Universitário Unieuro

ISSN: $1809-1261$

UNIEURO, Brasília, número 25 (Especial), 2018, pp. 7-25.

47. Trotta M.,Peira E., Carlotti M.E., Gallarate M. Deformable lipososomes for dermal administration of methotrexate, In International Journal of Pharmaceutics, 2004, vol. 270, pp. $119-125$.

48. Mishura D., Garg M., ubey V., Jain S., Jain N.K. Elastic Liposomes Mediated Transdermal Delivery of an Anti-Hypertensive Agent: Propanolo Hydrochloride. In Journal of Pharmacentical Science, 2007, vol. 94 (1), pp. 145-155.

49. Parhi R. and Padilama Suresh P., Production of Solid Lipid Nanoparticles-Drug Loading and Release Mechanism, In J. Chem. Pharm. Res., 2010, vol.2(1), pp. 211-227.

50. Mehnert, W., Mäder, K.. Solid lipid nanoparticles: production, characterization and applications. In Advanced Drug Delivery Reviews, 2001, vol. 47, no. 2-3, pp.165-96.

51. Rai, S.; Paliwal, R., Gupta, P. N.; Khatri, K.; Goyal, A. K.;Vaidya, B.; Vyas, S. P. Solid Lipid Nanoparticles (SLNs) as a Rising Tool in Drug Delivery Science: One Step Up in Nanotechnology. In Curr. Nanosci. 2008, vol.4, pp. 30-44.

52. Bleve M., Pavanetto F. and Perugini P., Solid Lipid Nanoparticles: Technological Developments and in Vivo Techniques to Evaluate Their Interaction with the Skin, In Progress in Molecular and Environmental Bioengineering - From Analysis and Modeling to Technology Applications, 2011, Capitúlo 8. Prof. Angelo Carpi (Ed.), ISBN: 978-953-307-268-5, www.intechopen.com.

53. Williams, A.C., Barry, B.W., The enhancement index concept applied to terpene penetration enhancers for human skin and model lipophilic (oestradiol) and hydrophilic (5-fluorouracil) drugs. In Int. J. Pharm.,1991, vol. 74, pp.157- 168.

54. Müller, R.H., Radtke, M., Wissing, S.A. (2002). Solid lipid nanoparticles (SLN) and nanostructured lipid carriers (NLC) in cosmetic and dermatological preparations, In Advanced Drug Delivery Reviews, 2002, vol. 54, n. 1, pp. 131-55.

55. Schroeter, A. (2010). New nanosized technologies for dermal and transdermal drug delivery. A review. In Journal of biomedical nanotechnology, 2010, vol. 6, n. 5, pp. 511-28.

56. Prausnitz, M.R. and Langer, R., Transdermal Drug Delivery, In Nature Biotechnology, 2008, vol. 26(11), pp. 1261-1268.

57. Honeywell-Nguyen, P.L., Arenja, S., Bouwstra, J.A., 2003. Skin penetration and mechanisms of action in the delivery of the D2-agonist rotigotine from surfactant-based elastic vesicle formulations, In Pharm. Res., 2003, vol. 20, pp.1619-1625. 
Artigo original

Hegemonia - Revista Eletrônica do Programa de Mestrado em Direitos Humanos,

Cidadania e Violência/Ciência Política do Centro Universitário Unieuro

ISSN: $1809-1261$

UNIEURO, Brasília, número 25 (Especial), 2018, pp. 7-25.

58. Honeywell-Nguyen, P.L., Bouwstra, J.A., The in vitro transport of pergolide from surfactant-based elastic vesicles through human skin: a suggested mechanism of action, In J. Control. Release, 2003, vol.86, pp.145-156.

59. H. Hillaireau, P. Couvreur, Nanocarriers' entry into the cell: relevance to drug delivery, In Cell. Mol. Life Sci., 2009, vol. 66, pp. 2873-2896.

60. F. Danhier, E. Ansorena, J.M Silva, et al., PLGA-based nanoparticles: An overview of biomedical applications, In J. Control. Release, 2012, vol. 161(2), pp. 505-12.

61. Couvreur P. Polyalkylcyanoacrylates as colloidal drug carriers. In Crit Rev Ther Drug Carr Syst., 1988, vol. 5, pp.1-20.

62. Prasad Rao J., Kurt E. Geckelera Polymer nanoparticles: Preparation techniques and size-control Parameters, In Progress in Polymer Science, 2011, vol.36 (7), pp. 887-913

63. Nazarenus M., Qian Zhang, Mahmoud G. Soliman, Pablo del Pino, et. al.,. In vitro interaction of colloidal nanoparticles with mammalian cells: What have we learned thus far?, In Beilstein J. Nanotechnol., 2014, vol. 5, pp. 1477-1490.

64. Bhakta G., Kumar Sharma R., Gupta N., Cool S., et.al., Multifunctional silica nanoparticles with potentials of imaging and gene delivery. In Nanomedicine: Nanotechnology, Biology, and Medicine, 2011, vol.7, pp.472-479.

65. Gref R., Couvreur P., Barratt G., and Mysiakine E., Surface-engineered nanoparticles for multiple ligand coupling, In Biomaterials, 2003, vol. 24, pp.4529-4537.

66. Gref R., Rodrigues J. and Couvreur P. Polysaccharides Grafted with Polyesters: Novel Amphiphilic Copolymers for Biomedical Applications, In Macromolecules, 2002, vol. 35, pp. 9861-9867.

67. Fonseca C. Simões, S. Gaspar R., Paclitaxel-loaded PLGA nanoparticles: preparation, physiochemical characterization and in vitro anti-tumoral activity, In J. Control. Release, 2002, vol. 83, pp. 273-286.

68. Garinot M., Fievez V., Pourcelle V., Stoffelbach F., des Rieux A., et. al., PEGylated PLGA-based nanoparticles targeting M cells for oral vaccination, In J. Control. Release, 2007, vol.120, pp. 195-204.

69. Couvreur P, Dubernet C, Puisieux F. Controlled drug delivery with nanoparticles: current possibilities and future trends. In Eur J Pharm Biopharm, 1995, vol.41, pp. 2-13. 
Artigo original

Hegemonia - Revista Eletrônica do Programa de Mestrado em Direitos Humanos,

Cidadania e Violência/Ciência Política do Centro Universitário Unieuro

ISSN: 1809-1261

UNIEURO, Brasília, número 25 (Especial), 2018, pp. 7-25.

70. Wang, S., Liu, W., Han, B., \& Yang, L. (2009). Study on a hydroxypropyl chitosangelatin based scaffold for corneal stroma tissue engineering. In Applied Surface Science, 2009, vol. 255, pp.8701-8705.

71. Arvanitoyannis, I. S., Totally and partially biodegradable polymer blends on natural and synthetic macromolecules: Preparation, physical properties, and potential as food packing materials. In Journal of Macromolecular Science - Reviews in Macromolecular Chemistry and Physics, 1999, vol.39, pp. 205-258.

72. Sezer A.D., Cevher E. Topical drug delivery using chitosan nano- and microparticles. In Expert Opin Drug Deliv., 2012, vol. 9(9), pp. 1129-46.

73. Budhian A., Steven J. Siegel, Karen I. Winey, Haloperidol-loaded PLGA nanoparticles: Systematic study of particle size and drug content, In International Journal of Pharmaceutics, 2007, vol. 336, pp.367-375.

74. Javadzadeh Y., Ahadi F., Davaran S., Mohammadi G., Sabzevari A., Adibkia K., Preparation and physicochemical characterization of naproxen-PLGA nanoparticles, In Colloids and Surfaces B: Biointerfaces, 2010, vol. 81, pp. 498-502.

75. K. Ladewig, Drug delivery in soft tissue engineering, In Expert. Opin. Drug Deliv., 2011, vol. 9, pp. 1175-1188.

76. Berthet M., Gauthier Y., Céline Lacroix C., Verrier B., Monge, C., Nanoparticle-Based Dressing: The Future of Wound Treatment? In Trends in Biotechnology, 2017, vol. 35 (8), p. 771.

77. Brannon-Peppas L. Preparation and characterization of crosslinked hydrophilic networks, In Studies in Polymer Science, 1990, vol. 8, pp. 45-66.

78. Karen Reimer, P.M. Vogt, Bianca Broegmann, J. Hauser O., et. al., An Innovative Topical Drug Formulation for Wound Healing and Infection Treatment: In vitro and in vivo Investigations of a Povidone-Iodine Liposome Hydrogel. In Dermatology, 2000, vol. 201, pp. 235-241.

79. Thirumaleshwar S., Parthasarthi K. Kulkarni and Devegowda V. Gowda, Liposomal Hydrogels: A Novel Drug Delivery System for Wound Dressing, In Current Drug Therapy, 2012, vol. 7, pp. 212-218.

80. Cortivo R, Vindigni V, Iacobellis L, Abatangelo G, et. al., Nanoscale particle therapies for wounds and ulcers. In Nanomedicine, 2010, vol. 5 (4), pp. 641-56. 
Hegemonia - Revista Eletrônica do Programa de Mestrado em Direitos Humanos,

Cidadania e Violência/Ciência Política do Centro Universitário Unieuro

ISSN: 1809-1261

UNIEURO, Brasília, número 25 (Especial), 2018, pp. 7-25.

81. Kalashnikova, I.; Das, S.; Seal, S. Nanomaterials for wound healing: scope and advancement. In Nanomedicine, 2015, vol.10, pp. 2593-2612.

82. Korrapati, P. S.; Karthikeyan, K.; Satish, A.; Krishnaswamy, et. al., Recent advancements in nanotechnological strategies in selection, design and delivery of biomolecules for skin regeneration. In Mater. Sci. Eng., 2016, vol. 67, pp. 747-765.

83. Archana, D.; Dutta, J.; Dutta, P. K. Evaluation of chitosan nano dressing for wound healing: Characterization, in vitro and in vivo studies., In Int. J. Biol. Macromol. 2013, vol. 57, pp. 193-203.

84.Patrulea, V.; Ostafe, V.; Borchard, G.; Jordan, O. Chitosan as a starting material for wound healing applications. In Eur. J. Pharm. Biopharm. 2015, vol. 97, pp.417-426. 